The author reported no conflicts of interest.

The Journal policy requires editors and reviewers to disclose conflicts of interest and to decline handling or reviewing manuscripts for which they may have a conflict of interest. The editors and reviewers of this article have no conflicts of interest.

6. Choi JO, Daly RC, Lin G, Lahr BD, Wiste HJ, Beaver TM, et al. Impact of surgical ventricular reconstruction on sphericity index in patients with ischaemic cardiomyopathy: follow-up from the STICH trial. Eur J Heart Fail. 2015;17: 453-63.

https://doi.org/10.1016/j.jtcvs.2020.06.136

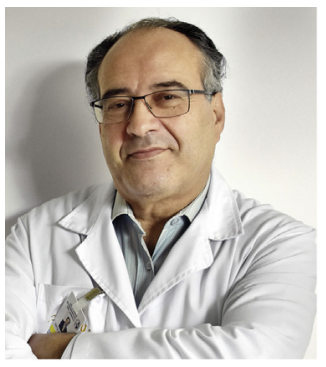

\section{REPLY: THE BAD} GUY: LEFT

\section{VENTRICULAR FUNCTION, SIZE, OR BOTH?}

\section{Reply to the Editor:}

In their article recently published in the Journal, Omer and colleagues ${ }^{1}$ demonstrated that in patients subjected to isolated coronary artery bypass grafting (CABG), depressed left ventricular ejection fraction (LVEF) was strongly associated with incremental rates of some complications (ie, renal failure, prolonged ventilation, and mechanical support), leading to increased mortality. They also found that the risk of specific complications may vary by LVEF class according to other preoperative characteristics (ie, preexisting renal failure or lung disease). They concluded that "prevention and effective treatment of complications should remain a focus of quality improvement initiatives, and future work is needed to mitigate their long-term detrimental impact on survival."

In this issue of the Journal, in a letter to the editor, Fukunaga and $\mathrm{RaO}^{2}$ draw our attention to a study from their group, published almost simultaneously in another journal, which "revealed that there was no statistically significant difference in terms of mortality, major morbidity, and prolonged hospital stay between patients with LVEF $<20 \%$ and LV size $<5.4 \mathrm{~cm}$ and those with preserved LVEF who underwent isolated CABG." In contrast, the combination of poor LV function and significant dilatation led to significantly worse outcomes. Thus, Fukunaga and Rao conclude that "the long-term effect of perioperative complications might indeed be greater in patients with LV dysfunction and an enlarged LV."
The author reported no conflicts of interest.

The Journal policy requires editors and reviewers to disclose conflicts of interest and to decline handling or reviewing manuscripts for which they may have a conflict of interest. The editors and reviewers of this article have no conflicts of interest.

Obviously, the 2 studies are not comparable; Omer and colleagues ${ }^{1}$ analyze the Veterans' Administration database involving more than 61,000 patients, including 1707 with LVEF $<25 \%$, which may be a close representation of the real world, whereas Fukunaga and Rao conducted an institutional review of a much smaller cohort (143 patients with LVEF $<20 \%$ ), which reflects the known expertise of a single institution. Nonetheless, importantly, the latter study stresses the fact that low LVEF per se is not the devil; rather, its association with ventricular dilation appears to be the reason for the poorer results. In my interpretation, low LVEF without ventricular dilation may be reversible, whereas a dilated ventricle represents a chronic phase, usually irreversible. And that makes the difference.

In an invited commentary accompanying the article by Omer and colleagues, I stressed that the relationship between preoperative comorbidities (eg, preexisting renal failure, lung disease, uncontrolled diabetes), especially when in association, and the immediate and late mortality of cardiac surgical procedures is well known. "Adding myocardial insufficiency to the 'cocktail' can only make it worse," meaning that the problem does not simply lie in the function or size of the left ventricle. Again, in my view, the main importance of the work by Omer and colleagues lies in the assumption that we can do something about reducing the number and severity of complications, thereby decreasing mortality, by modulating risk factors and modifying surgical methods and techniques. As the authors state, "prevention and effective treatment of complications should remain a focus of quality improvement initiatives, and future work is needed to mitigate their long-term detrimental impact on survival."

Manuel J. Antunes, $M D, P h D, D S c$ Faculty of Medicine Clinic of Cardiothoracic Surgery University of Coimbra Coimbra, Portugal

\section{References}

1. Omer S, Adeseye A, Jimenez E, Cornwell LD, Massarweh NN. Low left ventricular ejection fraction, complication rescue, and long-term survival after coronary artery bypass grafting. J Thorac Cardiovasc Surg. March 6, 2020; https://doi.org/ 10.1016/j.jtcvs.2020.03.040 [Epub ahead of print]. 